BULL. AUSTRAL. MATH. SOC.

VOL. $4(1971), 35-39$.

\title{
Minimal vector lattice covers
}

\section{Paul F. Conrad}

We show that each abelian $l$-group $G$ is a large $Z$-subgroup of a minimal vector lattice $V$ and if $G$ is archimedean then $V$ is unique, in fact, $V$ is the 2 -subspace of $\left(G^{d}\right)^{\wedge}$ that is generated by $G$, where $G^{d}$ is the divisible hull of $G$ and $\left(G^{d}\right)^{\wedge}$ is the Dedekind-MacNeille completion of $G^{d}$. If $G$ is non-archimedean then $V$ need not be unique, even if $G$ is totally ordered.

Throughout this note group will always mean abelian group. An Z-subgroup $A$ of an $Z$-group $B$ is lorge if for each $Z$-ideal $L \neq 0$ of $B, L \cap A \neq 0$ (or equivalently each $Z$-homomorphism of $B$ that is one to one on $A$ is an isomorphism). In this case we shall also call $B$ an essential extension of $A$.

We define $U$ to be a $v$-hulz of an $Z$-group $G$ if

i) $U$ is a vector lattice and $G$ is a large $Z$-subgroup of $U$, and

ii) no proper $Z$-subspace of $U$ contains $G$.

Note that $U$ contains a copy of $G^{d}$ and since $G^{d}$ is divisible and large in $U$ it is dense in $U$ (that is, $0<u \in U$ implies $0<g<u$ for some $\left.g \in G^{d}\right)$. Thus ([1], p. 116), the infinite joins and intersections that exist in $G$ agree with those in $U$.

PROPOSITION. Each $z$-group $G$ admits a v-hull. Thus if $G$ is an Z-subgroup of a unique minimal vector lattice $U$ then $U$ must be a

Received 22 August 1970. 
$v-h u z z$ of $G$.

Proof. $G$ is a subdirect sum of o-groups and by the Hahn representation theorem, ([4], p. 59), each o-group can be embedied in a vector lattice of real valued functions. Thus we may assume that $G$ is an $Z$-subgroup of a vector lattice $V$. Let $W$ be the intersection of all the Z-subspaces of $V$ that contain $G$. Let $B$ be an $Z$-ideal of $W$ that is maximal with respect to $B \cap G=0$. Then

$$
G \cong(B \oplus G) / B \subseteq W / B
$$

and this is an essential extension. For if $U$ is an $Z$-ideal of $W$ that contains $B$ and $U / B \cap(B \oplus G) / B=B$, then $B=U \cap(B+G)=B+(U \cap G)$ and so $U \cap G \subseteq B$. Thus $U \cap G=0$ and $U \supseteq B$ and so $U=B$. Therefore $W / B$ is a $v$-hull of $(B \oplus G) / B$.

THEOREM. An archimedean Z-group $G$ admits a unique v-hulz. This $v$-hull is $\left(Z\right.$-isomorphic to the 2 -subspace of $\left(G^{d}\right)^{\wedge}$ that is generated by $G$ and hence it is archimedean.

Proof. Let $U$ be a $v$-hull of $G$.

(1) $U$ is archimedean. For suppose by way of contradiction that $0<a, b \in U$ and $n a<b$ for all integers $n>0$. Since $G$ is large in $U$ there exists $0<x \in G$ such that $x<m a$ for some $m>0$. Since $U$ is minimal,

$$
U=\bigvee U(g) \text { for all } g \in G^{+} \text {, }
$$

where $U(g)$ is the $Z$-ideal of $U$ that is generated by $g$. Thus $b=b_{1}+\ldots+b_{t}$, where $b_{i} \in U\left(g_{i}\right)$ for $i=1, \ldots, t$ and hence

$$
b=\left|b_{1}+\ldots+b_{t}\right| \leq\left|b_{1}\right|+\ldots+\left|b_{t}\right| \text {. }
$$

Now there exists $k>0$ such that $b_{i} \leq k g_{i}$ for $i=1, \ldots, t$. Thus $b \leq k\left(g_{1}+\ldots+g_{t}\right)=y \in G$ and hence

$$
n x<n m a<b \leq y \text { for all } n>0 \text {; }
$$

but this contradicts the fact that $G$ is archimedean.

(2) $U$ is unique. Since $U$ is divisible we may assume that $G^{d}$ is 
an 2 -subgroup of $U$. Thus $G^{d}$ is dense in $U$ and hence, (see [2]), $\left(G^{d}\right)^{\wedge}$ is the 2 -ideal of $U^{\wedge}$ that is generated by $G^{d}$ and so $\left(G^{d}\right)^{\wedge}$ is an $\tau$-subspace of $U^{\wedge}$ that contains $G^{d}$. Also since $U^{\wedge}$ is archimedean it follows that $U$ is an Z-subspace of $U^{\wedge}$, (see [3]). Thus

$$
G \subseteq G^{d} \subseteq U \subseteq\left(G^{d}\right)^{\wedge}
$$

and so $U$ is the $Z$-subspace of $\left(G^{d}\right)^{\wedge}$ that is generated by $G$ and hence it is unique.

COROLLARY I. If $U$ and $V$ are $v$-huzzs of an archimedean 2 -group $G$ then there exists a unique $z$-isomorphism $\pi$ of $U$ onto $V$ such that $g \pi=g$ for $a z z \quad g \in G$.

Proof. This is true for $G^{d}$ and for $\left(G^{d}\right)^{\wedge}$.

COROLLARY II. If $G$ is a subdirect sim of reals then its $v$-huzl is contained in this sum of reals.

$$
\text { Proof. If } G \subseteq \prod R_{i} \text {, then }\left(G^{d}\right)^{\wedge} \subseteq \prod R_{i} \text {, (see [2]). }
$$

If $A$ is an 2 -subgroup of an 2 -group $B$ then $B$ is an a-extension of $A$ if $L \rightarrow L \cap A$ is a one to one mapping of the $Z$-ideals of $B$ onto the $z$-ideals of $A$ (or equivalently for each $0<b \in B$ there exist $0<a \in A$ and a positive integer $n$ such that $a<n b$ and $b<n a$ ).

PROPOSITION. Each $v$-hulz of an o-group $G$ is an o-group, but it need not be an a-extension. An o-group $G$ admits a v-hull that is an a-extension, but it need not be unique.

Proof. If $H$ is an essential extension of $G, 0<a, b \in H$ and $a \wedge b=0$ then there is a positive integer $n$ and $0<x, y \in G$ such that $n a>x$ and $n b>y$. Thus $0=n(a \wedge b)=n a \wedge n b \geq x \wedge y \geq 0$ which contradicts the fact that $G$ is an o-group. Thus $H$ is totally ordered and so each $v$-hull of $G$ is an o-group.

Let $\quad U=R \oplus R \oplus R$ lexicographically ordered from the left and let $G$ be the subgroup of $U$ generated by $(0,0,1),(\pi, 1,0)$ and $(1, \pi, 0)$. Then each subspace $N$ of $U$ that contains $G$ must contain 
$\pi(\pi, 1,0)-(1, \pi, 0)=\left(\pi^{2}-1,0,0\right)$ and hence $N=U$. Therefore $U$ is a $v$-hull of $G$ but clearly not an a-extension since $U$ has two proper convex subgroups, but $G$ has only one.

REMARKS. Note that

$$
G \cong \text { (the subgroup of } R \text { generated by } \pi \text { and } 1 \text { ) } \oplus R
$$

and so $R \oplus R$ is a $v$-hull and an a-extension of $G$. If we let $U=R \oplus R$ lexicographically ordered from the left and let $G$ be the subgroup of $U$ generated by $(\pi, 1)$ and $(1, \pi)$ then $U$ is a minimal vector lattice containing $G$ but it is not an essential extension and so not a $v$-hull. Also, $G$ but not $U$ is archimedean.

Now by Hahn's representation theorem each $0-$ group $G$ can be embedded in a vector lattice $V$ that is an $a$-extension of $G$ and hence the intersection of all the subspaces of $V$ that contain $G$ is a $v$-hull and an $a$-extension of $G$.

$$
\text { Finally let } V=\prod_{i=1}^{\infty} R_{i} \text { lexicographically ordered from the left and }
$$

let $f$ be a group isomorphism of $R$ onto $\prod_{i=2}^{\infty} R_{i}$ such that

$f(1)=(1,0,0, \ldots)$ and in general $f(x)=\left(f_{2}(x), f_{3}(x), \ldots\right)$. Define

$$
\left(x_{1}, x_{2}, \ldots\right) \tau=\left(x_{1}, x_{2}+f_{2}\left(x_{1}\right), x_{3}+f_{3}\left(x_{1}\right), \ldots\right) .
$$

Then $\tau$ is an o-automorphism of $V$. For each $x \in V$ and $r \in R$ define

$$
r *(x \tau)=(r x) \tau
$$

Then $(V, *)$ is a vector lattice. Note that

$$
\begin{aligned}
r_{*}\left(x, f_{2}(x), f_{3}(x), \ldots\right) & =r_{*}((x, 0,0, \ldots) \tau) \\
& =(r x, 0,0, \ldots) \tau \\
& =\left(r x, f_{2}(r x), f_{3}(r x), \ldots\right) .
\end{aligned}
$$

In particular, for $x=1$ we have

$$
r_{*}(1,1, \ldots)=\left(r, f_{2}(r), f_{3}(r), \ldots\right) \text {. }
$$


Thus $(V, *)$ is a $v$-hull and an a-extension of the $l$-group $G=\sum_{i=1}^{\infty} R_{i}$ and $G$ is also a vector lattice with respect to the natural scalar multiplication and so $G$ is its own $v$-hull. But $G$ and $V$ are not o-isomorphic since $V$ is $a$-closed (that is, admits no proper a-extensions) and $G$ is not.

REMARK. If the chain of convex subgroups of an o-group $G$ satisfies the DCC then the Hahn group corresponding to $G$ is the unique $v$-hull of $G$ that is also an a-extension.

\section{References}

[1] S.J. Bernau, "Orthocompletion of lattice groups", Proc. London Math. Soc. (3) 16 (1966), 107-130.

[2] Paul Conrad and Donald McAlister, "The completion of a lattice ordered group", J. Austral. Math. Soc. 9 (1969), 182-208.

[3] Paul Conrad, "Free abelian Z-groups and vector lattices", Math. Ann. (to appear).

[4] L. Fuchs, Partially ordered algebraic systems (Pergamon Press, Oxford, London, New York, Paris, 1963).

University of Kansas,

Lawrence,

Kansas, USA. 